Supporting Information

ACS Chemical Neuroscience

\title{
The ionotropic glutamate receptor GluA2 in complex with bicyclic pyrimidinedione-based compounds: when small compound modifications have distinct effects on binding interactions
}

Karla Frydenvang ${ }^{1}$, Darryl S. Pickering ${ }^{1}$, Giridhar U. Kshirsagar ${ }^{1,2}$, Giulia Chemi ${ }^{2, ¥}$, Sandra Gemma $^{2}$, Desiree Sprogøe ${ }^{1}$, Anne Mette Kærn ${ }^{1}$, Simone Brogi ${ }^{3 *}$, Giuseppe Campiani ${ }^{2}$, Stefania Butini $^{2, *}$, Jette Sandholm Kastrup ${ }^{1, *}$

${ }^{1}$ Department of Drug Design and Pharmacology, University of Copenhagen, Jagtvej 162, DK-2100 Copenhagen, Denmark

${ }^{2}$ Department of Biotechnology, Chemistry and Pharmacy, (DoE 2018-2022), University of Siena, Via A. Moro 2, 53100 Siena, Italy

${ }^{3}$ Department of Pharmacy, University of Pisa, Via Bonanno 6, 56126 Pisa, Italy

¥present address: Wellcome Centre for Anti-Infectives Research, Drug Discovery Unit, Division of Biological Chemistry and Drug Discovery, University of Dundee, DD1 5EH Dundee, United Kingdom

\section{Table of contents:}

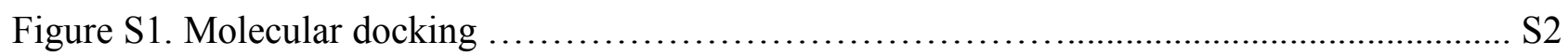

Table S1. Ligand-binding energies $(\mathrm{kcal} / \mathrm{mol})$ for compound 3-7 ............................................. S3 

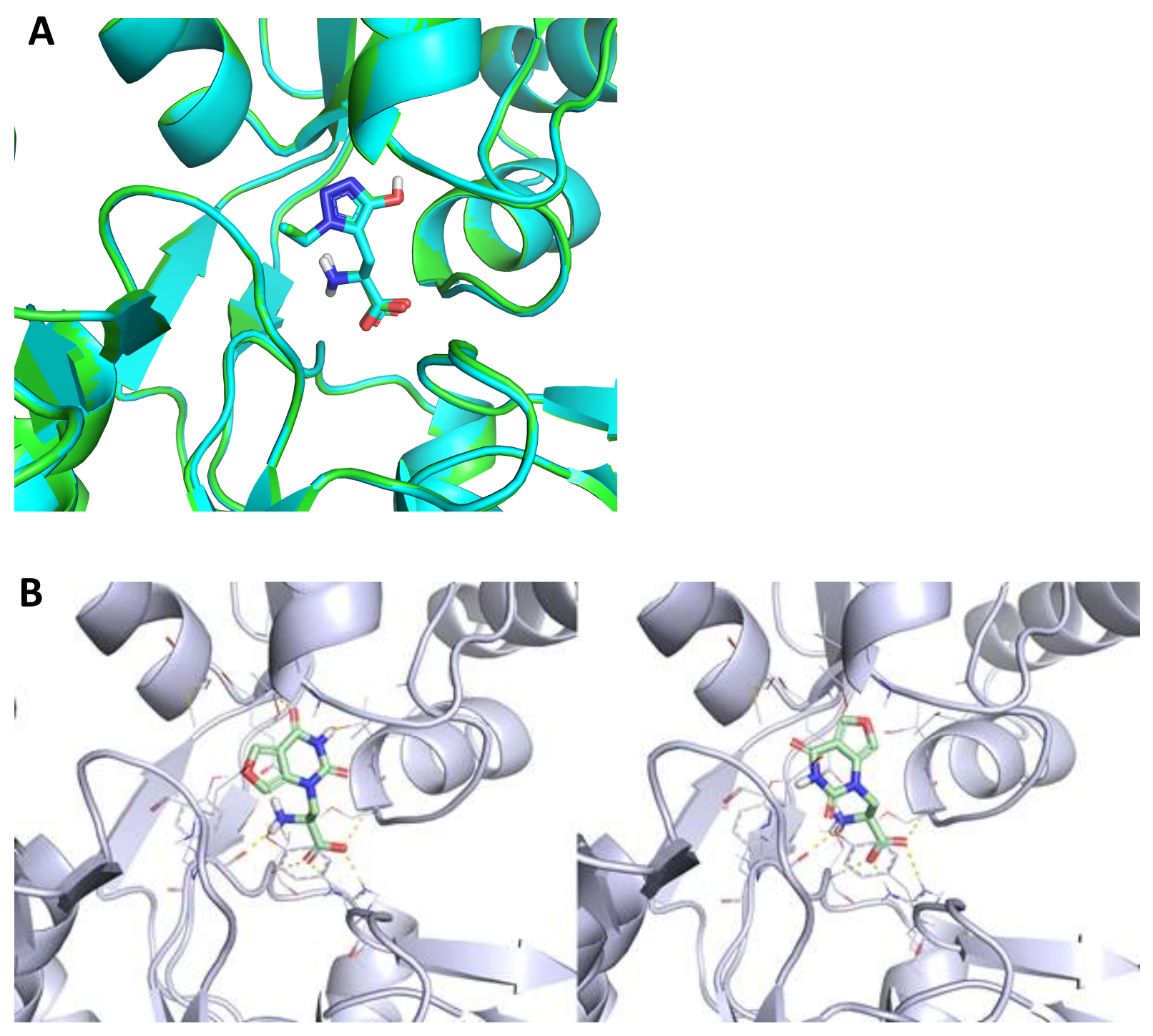

Figure S1. Molecular docking. (A) Superposition of the crystal structure of GluA2-LBD with HJ8 (PDB code 6Q54; represented in cyan cartoon and sticks, respectively) and the redocked pose of the same ligand (represented in green cartoon and sticks, respectively). (B) Docked poses of compound 7 (pale green sticks) into the crystal structure of GluA2-LBD (light grey cartoon) crystallized in complex with 7. 
Table S1. Ligand-binding energies (kcal/mol) for compounds 3-7 docked into GluA2-LBD (PDB code 6Q54).

\begin{tabular}{|l|l|}
\hline Compound & $\begin{array}{l}\Delta \mathbf{G}_{\text {bind }} \\
(\mathbf{k c a l} / \mathbf{m o l})\end{array}$ \\
\hline $\mathbf{3}$ & -51.0 \\
\hline $\mathbf{4}$ & -50.5 \\
\hline $\mathbf{5}$ & -50.0 \\
\hline $\mathbf{6}$ & -50.2 \\
\hline $\mathbf{7}$ & -53.5 \\
\hline $\mathbf{7}$ turned & -36.3 \\
\hline
\end{tabular}

Footnote: For compounds 3-6 the reported clusters showed a percentage of docked solutions over $97 \%$. This means that the contribution to the binding affinity for compounds 3-6 is ascribable only to one cluster (X-ray pose) as the clusters with turned or non-canonical poses are under $3 \%$ of the docked solutions. On the contrary, for compound 7 we found two possible clusters (X-ray and turned) as both of them present docked solutions in percentage over 30\%. Accordingly, both clusters were taken into consideration for the binding affinity, leading to a reduction in the prediction of the binding affinity of 7 . 